\title{
Noradrenergic Activation Amplifies Bottom-Up and Top-Down Signal-to-Noise Ratios in Sensory Thalamus
}

\author{
Akio Hirata, Juan Aguilar, and Manuel A. Castro-Alamancos \\ Department of Neurobiology and Anatomy, Drexel University College of Medicine, Philadelphia, Pennsylvania 19129
}

\begin{abstract}
Thalamocortical cells receive sensory signals via primary sensory afferents and cortical signals via corticothalamic afferents. These signals are influenced by a variety of neuromodulators that are released in the thalamus during specific behavioral states. Hence, different neuromodulators may set different thalamic modes of sensory information processing. We found that noradrenergic activation affects sensory and corticothalamic signals in the whisker thalamus differently than cholinergic activation. Whereas cholinergic activation increases the spontaneous firing (noise) and enlarges the receptive fields of ventroposterior medial thalamus (VPM) cells, noradrenergic activation decreases spontaneous firing and focuses receptive fields. Consequently, for sensory signals, noradrenergic activation sets bottom-up thalamic processing to a focused and noise-free excitatory receptive field, which contrasts with the broad and noisy excitatory receptive field characteristic of cholinergic activation. For corticothalamic signals, noradrenergic activation sets top-down processing to a noise-free high-frequency signal detection mode, whereas cholinergic activation produces a noisy broadband signal detection mode. The effects of noradrenergic activation on signal-to-noise ratios of VPM cells were found to be mediated by nucleus reticularis thalamic (nRt) cells. Hence, a major role of $n R t$ cells is to regulate the noise level of thalamocortical cells during sensory processing. In conclusion, different modulators establish distinct modes of bottom-up and top-down information processing in the sensory thalamus.
\end{abstract}

Key words: thalamus; vibrissas; sensory processing; vigilance; attention; locus ceruleus; brainstem reticular formation; acetylcholine; norepinephrine; corticothalamic

\section{Introduction}

Thalamocortical cells are at the center of a neuronal network that involves sensory, cortical, and modulatory inputs (Sherman and Guillery, 1996). In the ventroposterior medial thalamus (VPM), thalamocortical cells form clusters, called barreloids (Land et al., 1995), that project to clusters of cells in layer IV of somatosensory cortex, called barrels (Woolsey and Van der Loos, 1970). VPM cells receive signals from four main sources. Primary sensory fibers originating from clusters of cells, called barrelettes (Henderson and Jacquin, 1995), in the principal trigeminal nucleus provide sensory (bottom-up) signals. Corticothalamic fibers originating in layer VI of barrel cortex (Bourassa et al., 1995) provide top-down influences. Fibers from nucleus reticularis thalamic (nRt) cells (Ohara and Lieberman, 1985) provide the main inhibitory control in VPM. Finally, a variety of neuromodulator fibers originate mainly in several brainstem nuclei. Among them, cholinergic fibers derive from laterodorsal tegmentum (LDT) or pedunculopontine (PPT) nuclei (Satoh and Fibiger, 1986; Hallanger et al., 1987), and noradrenergic fibers originate in the locus ceruleus (LC) (Lindvall et al., 1974; Simpson et al., 1997).

\footnotetext{
Received Dec. 12, 2005; revised March 19, 2006; accepted March 20, 2006.

This work was supported by the National Institutes of Health and the EJLB Foundation. We thank Yoshie TawaraHirata for excellent technical support.

Correspondence should be addressed to Dr. Manuel A. Castro-Alamancos, Department of Neurobiology and Anatomy, Drexel University College of Medicine, 2900 Queen Lane, Philadelphia, PA 19129. E-mail: mcastro@drexelmed.edu.

DOI:10.1523/JNEUROSCI.5298-05.2006

Copyright $\odot 2006$ Society for Neuroscience $\quad$ 0270-6474/06/264426-11\$15.00/0
}

Early studies in several sensory systems recognized the impact of arousal and behavioral state on the responsiveness and activity of thalamic neurons to sensory inputs (Poggio and Mountcastle, 1963; Livingstone and Hubel, 1981; Swadlow and Weyand, 1985) (for review, see Castro-Alamancos, 2004b). These dynamic changes are primarily caused by neuromodulators released in thalamus during specific behavioral states (Vanderwolf, 1988; Steriade and McCarley, 1990; Jones, 1993). In particular, cholinergic neurons in the LDT/PPT complex discharge vigorously during paradoxical sleep and also during wakefulness (el Mansari et al., 1989; Steriade et al., 1990), and the levels of acetylcholine increase in the thalamus during those states (Williams et al., 1994). Noradrenergic neurons in the LC discharge robustly during high levels of vigilance and attention, reduce their firing during slow-wave sleep, and stop firing during paradoxical sleep (Hobson et al., 1975; Foote et al., 1980; Aston-Jones and Bloom, 1981; Aston-Jones et al., 1991), and their firing produces forebrain activation (Berridge and Foote, 1991). Because both systems, cholinergic and noradrenergic, are active during arousal and induce forebrain activation (Moruzzi and Magoun, 1949), it is particularly relevant to know whether they have similar or, instead, selective effects on thalamocortical information processing.

Many studies have investigated the effects of electrical stimulation of cholinergic (LDT/PPT) and noradrenergic (LC) brainstem nuclei on thalamocortical network responses. However, these effects are difficult to interpret because these nuclei are intricately interconnected between each other and project to ar- 
eas that also innervate the thalamus. Thus, LC stimulation may influence cholinergic cells in LDT/PPT or other thalamusprojecting cells. A different approach to study the selective effects of these neuromodulators is to infuse them directly into the thalamocortical network.

In the vibrissa system of rodents, cholinergic activation enlarges the receptive fields of VPM cells (Aguilar and CastroAlamancos, 2005) and enhances their responses to highfrequency sensory signals, virtually eliminating rapid sensory adaptation in the sensory thalamus (Castro-Alamancos, 2002a). However, the effects of noradrenergic activation on sensory responses of VPM cells are unknown. Moreover, the effects of either cholinergic or noradrenergic activation on the responses of VPM cells to cortical signals are also unknown. In the visual system, application of norepinephrine to lateral geniculate nucleus cells via iontophoresis enhances their firing and responsiveness to optic nerve electrical stimuli (Rogawski and Aghajanian, 1980a,b) and affects responses to visual stimuli (Funke et al., 1993).

In the present study, we compared the effects of cholinergic and noradrenergic thalamic activation on the responses of VPM cells to sensory and cortical signals in urethane-anesthetized rats. The results show that these modulators have highly selective effects that set different modes of sensory and corticothalamic information processing.

\section{Materials and Methods}

Surgery. Sixty adult Sprague Dawley rats (300-350 g) were used in this study and cared for in accordance with National Institutes of Health guidelines for laboratory animal welfare. All experiments were approved by the Drexel University Institutional Animal Care and Use Committee. Rats were anesthetized with urethane $(1.5 \mathrm{~g} / \mathrm{kg}$, i.p.) and placed in a stereotaxic frame. All skin incisions and frame contacts with the skin were injected with lidocaine (2\%). A unilateral craniotomy extended over a large area of the parietal cortex. Small incisions were made in the dura as necessary. Body temperature was automatically maintained constant with a heating pad at $37^{\circ} \mathrm{C}$. The level of anesthesia was monitored with field recordings and limb-withdrawal reflexes and kept constant at approximately stage III using supplemental doses of urethane.

Electrophysiology. Single-unit recordings in VPM were performed, as described previously (Castro-Alamancos, 2002a), using electrodes pulled from glass pipettes $(10-30 \mathrm{M} \Omega)$ that were filled with saline. These electrodes generally record only a well discernible single-unit of very large amplitude ( $>10$ times the noise). Every cell included in this study corresponds to a recording in which there was only one discernable largeamplitude spike in the recording electrode. Also, a tungsten electrode was placed in the depth $(0.5-1 \mathrm{~mm})$ of the frontal agranular (motor) or barrel neocortex to record spontaneous field potential activity (filter settings, $0.7-170 \mathrm{~Hz}$ ) and thus to monitor the depth of anesthesia. This electrode was placed in the hemisphere contralateral to the stimulated whiskers.

Recordings from VPM contralateral to the stimulated whiskers were at approximately the following coordinates (Paxinos and Watson, 1982) from bregma (in mm): posterior, 3.5 ; lateral, 3; depth, 5-6. Coordinates for $\mathrm{nRt}$ cells were the same, but electrodes were more lateral $(\sim 4 \mathrm{~mm})$. The electrode used to stimulate corticothalamic fibers in the thalamic radiation was placed at approximately (in $\mathrm{mm}$ ): posterior, 2.5; lateral, 4.5 ; depth, 3.5-5. In every case, the thalamic radiation stimulating electrode and the VPM recording electrode were diligently aligned so that stimulation of the thalamic radiation resulted in virtually no response at low frequency but a highly reliable $(\sim 100 \%)$ response at $5-40 \mathrm{~Hz}$ of short latency $(<7 \mathrm{~ms})$. The intensity was kept $<150 \mu \mathrm{A}$.

Whisker and thalamic radiation stimulation. Sensory stimulation consisted of independently deflecting up to six individual whiskers using six different whisker stimulators. After isolating a unit, the whiskers were stimulated using a hand-held probe. The whisker that produced the shortest latency and strongest response was considered the principal whisker (PW). This whisker and up to five additional whiskers surrounding it, called adjacent whiskers (AWs), were selected for stimulation. Each of them was placed in an independent whisker stimulator. Thus, in many cases, six whisker stimulators were used simultaneously, which allowed to independently stimulate the six whiskers. Each of the selected whiskers was inserted into a glass micropipette ( $1 \mathrm{~mm}$ diameter) that was glued to the membrane of a miniature speaker. Application of a $1 \mathrm{~ms}$ square current pulse to the speaker deflected the micropipette and the whiskers inside $(\sim 400 \mu \mathrm{m})$. The whisker stimulators were oriented in the preferred direction to produce the largest response as determined with the hand probe. Each of the six whisker stimulators were controlled by a computer-programmable Master-8 (A.M.P.I., Jerusalem, Israel).

Whisker and corticothalamic stimulation was delivered according to the following protocols. For whisker stimulation, a trial consisted of an initial $2 \mathrm{~s}$ without whisker stimulation, followed by stimulation delivered to each whisker at $2 \mathrm{~s}$ intervals (the order of whisker stimulation was randomly selected). The first whisker was stimulated $2 \mathrm{~s}$ after the trial began, the second whisker was stimulated $4 \mathrm{~s}$ after the trial started, and so on, so that the sixth (last) whisker stimulus was delivered $12 \mathrm{~s}$ after the start of the trial. Thus, a single trial contained stimuli for all six whiskers and lasted a total of $14 \mathrm{~s}$. Whisker stimuli consisted of four stimuli delivered at $2 \mathrm{~Hz}$ or 10 stimuli delivered at 10 or $20 \mathrm{~Hz}$. To afford time, only four stimuli were used at $2 \mathrm{~Hz}$ because little more adaptation is produced at this frequency with additional stimuli. For high-frequency whisker stimulation (e.g., $10 \mathrm{~Hz}$ ) analyses, the last stimulus in the train was used.

For corticothalamic stimulation, a trial consisted of $3 \mathrm{~s}$ of no stimulation, followed by a 10 pulse train delivered at 2, 5, 10, 20, and $40 \mathrm{~Hz}$. Each trial lasted $11 \mathrm{~s}$. Each peristimulus time histogram (PSTH) was created with a minimum of 20 trials for corticothalamic stimulation and 30 trials for whisker stimulation using a $1 \mathrm{~ms}$ bin size.

Microdialysis. To apply drugs into VPM, a microdialysis cannula (250 $\mu \mathrm{m}$ diameter, 2-mm-long membrane) was placed around the following coordinates (in mm): posterior, 3; lateral, 2-3; depth, 4-6 as described previously (Castro-Alamancos, 2002a). The cannula entered into the brain at an angle $\left(\sim 30^{\circ}\right)$ from the midline, whereas the VPM recording electrode entered lateral to the cannula and parallel to the midline. Artificial CSF (ACSF) was continuously infused through the probe at 2-4 $\mu \mathrm{l} / \mathrm{min}$. ACSF contained the following (in $\mathrm{mm}$ ): $126 \mathrm{NaCl}, 3 \mathrm{KCl}, 1.25$ $\mathrm{NaH}_{2} \mathrm{PO}_{4}, 26 \mathrm{NaHCO}_{3}, 1.3 \mathrm{MgSO}_{4} 7 \mathrm{H}_{2} \mathrm{O}, 10$ dextrose, and $1 \mathrm{CaCl}_{2}$ $2 \mathrm{H}_{2} \mathrm{O}$. Carbachol was dissolved in the ACSF at $0.5-1 \mathrm{~mm}$. Norepinephrine was dissolved at $0.5-1 \mathrm{~mm}$ and protected from light and from oxidation with ascorbic acid. Atropine and phentolamine were used at $1-2$ mM. Bicuculline methiodide (BMI) and CGP35348 ( $p$-3-aminopropylp-diethoxymethyl phosphoric acid) (CGP) were used at 100-300 $\mu \mathrm{m}$ and 5-10 mM, respectively. The diffusion of the drugs using microdialysis was determined by placing the probe and an array of four recording electrodes at different distances from the probe. We found that norepinephrine $(1 \mathrm{~mm})$ and carbachol $(0.5 \mathrm{~mm})$ affected multiunit activity at distances of $0.5-1 \mathrm{~mm}$ but not at $1.5 \mathrm{~mm}$ or above from the probe. This indicates that the effects of the drugs are confined to a $1 \mathrm{~mm}$ area around the probe. This spread includes the VPM and nRt but may also affect parts of adjacent structures, such as the posterior nucleus and the zona incerta, both of which respond to whisker stimulation (Bartho et al., 2002; Trageser and Keller, 2004). Although potential effects mediated by these structures should be considered, we believe that these higher-order nuclei would be unlikely to explain most effects reported in the present paper.

Data analysis. Spontaneous cell firing was computed by counting the number of spikes during the $2-3 \mathrm{~s}$ period at the beginning of each trial and for a minimum of 20-30 trials. Population data are presented as mean \pm SD. Statistics for comparing population data between quiescent (control) and cholinergic or noradrenergic states consisted of paired $t$ tests if the populations were normal according to the Shapiro-Wilk normality test. Nonparametric tests consisted of Wilcoxon's signed-rank test for within-group comparisons and the Mann-Whitney test for between groups comparisons. Note that each cell is studied in at least two different states ( $n$ refers to number of cells).

Histology. At the end of the experiments, the animals were given an overdose of sodium pentobarbital and either perfused through the heart 
with saline followed by paraformaldehyde (4\%) or the brain was directly extracted and placed in the fixative. The brains were then sectioned in the coronal plane using a vibratome $(80-100 \mu \mathrm{m})$ and processed for Nissl staining. For the cells included in the study, subsequent analysis confirmed the location of electrode tracts within VPM and nRt.

\section{Results}

Dataset

VPM cells were identified by their short latency to a whisker deflection $(<7 \mathrm{~ms})$ and by tracing the track of the recording electrode in histological sections confirming that the electrode was inside VPM. The data shown in the present study are part of a growing dataset consisting of $>200$ well isolated single-unit thalamic recordings. Because the amplitude of these spikes is generally in the range of $5-10 \mathrm{mV}$, we presume that the electrode tip is very close to the membrane of the cell. Generally, these recordings are fairly stable so that the cells can be studied for several hours. All of the cells presented here are naive cells, unless otherwise indicated. That is, drug applications were done to one cell per animal.

Effect of noradrenergic activation on whisker-evoked responses in VPM cells To produce noradrenergic activation in the thalamus, we infused norepinephrine (0.5-1 mM) using microdialysis, as described previously (Castro-Alamancos, 2002a). To produce cholinergic activation, we infused carbachol (0.5-1 mM). In these experiments, up to six whisker stimulators were used simultaneously to map the responses of the PW and several AWs. An example of the effects of carbachol and norepinephrine on the same VPM cell is shown in Figure 1. During the quiescent (control) state, the spontaneous firing rate of this cell was $0.5 \mathrm{~Hz}$, whereas during carbachol, the firing rate increased to $6 \mathrm{~Hz}$, and during norepinephrine the cell had no spontaneous firing. PSTHs of responses to lowfrequency stimulation $(0.1 \mathrm{~Hz})$ of each of five individual whiskers revealed that, during the quiescent state, the cell responded primarily to two of the stimulated whiskers, the PW and one AW. However, during carbachol, the cell responded to stimulation of all five whiskers, albeit with different latencies and strengths. In contrast, during norepinephrine, the cell responded mostly to two of the five whiskers, with some responses present also for two other whiskers. Although cells responded exceedingly well to whisker stimuli during either carbachol or norepinephrine, during norepinephrine, the only activity present was that evoked by the whisker stimulus.

Figure $2 \mathrm{~A}$ shows population data of spontaneous firing and whisker- evoked responses from a group of cells that were subjected to either cholinergic $(n=8$ cells) or noradrenergic $(n=9)$ activation. The spontaneous firing was measured by counting the number of spikes during the $2 \mathrm{~s}$ period at the beginning of each for the 30 stimulation trials before the whisker stimulation was delivered (i.e., this corresponds to a $60 \mathrm{~s}$ period). This value is then converted to either spikes per second (in Hertz) or spikes per
$20 \mathrm{~ms}$ for comparison with the whisker-evoked responses. Whisker responses were measured as the spikes per stimulus during a $20 \mathrm{~ms}$ time window after the whisker deflection. The values during the control (quiescent) state for these cells were not significantly different for cells subjected to carbachol or norepinephrine, and thus they were averaged together in Figure $2 \mathrm{~A}$ for illustration purposes (control; $n=17$ ). However, all statistical comparisons are made within subjects by using the corresponding control values for each cell. As with the cell shown in Figure 1, all VPM cells increased their spontaneous firing during carbachol $(p<0.01)$ and all decreased their spontaneous firing during norepinephrine $(p<0.01)$. In fact, most cells had no spontaneous firing during norepinephrine. Despite the reduction of spontaneous firing during norepinephrine, the responses to whisker stimulation were robust. Figure $2 \mathrm{~A}$ illustrates whisker responses evoked by stimulating the PW and four AWs at low frequencies $(0.1$ and $2 \mathrm{~Hz})$ and at high frequencies $(10$ and $20 \mathrm{~Hz})$ during control states and during either cholinergic or noradrenergic activation. For low-frequency whisker stimulation, PW responses were robust during any of the three states, but AW responses were significantly stronger during carbachol than during the control state for all four AWs, indicating that the size of the receptive field had enlarged $(p<0.01)$. In contrast, during norepinephrine, only the first AW (i.e., the AW with the strongest response) produced a response that was significantly stronger than during the 
A

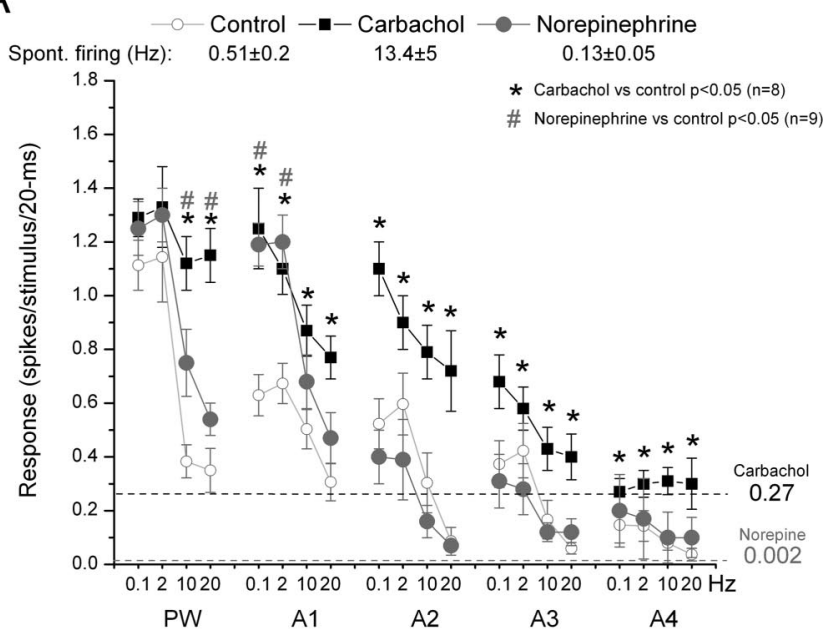

B

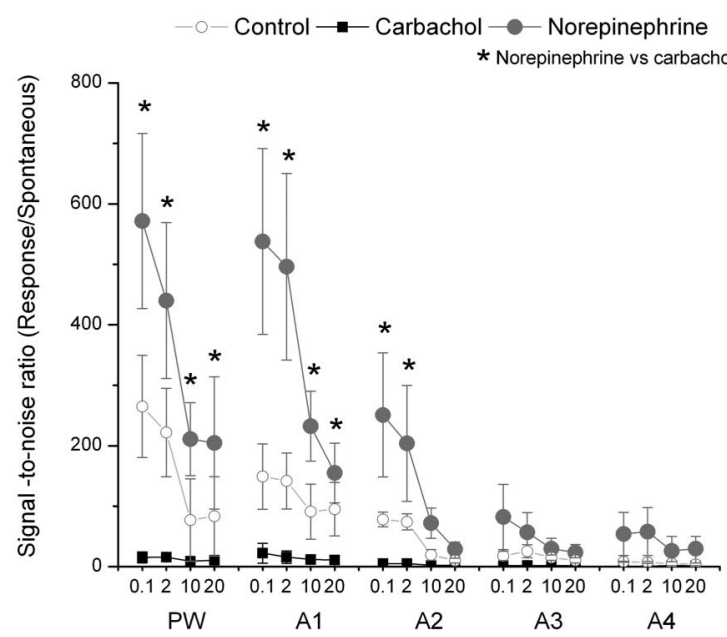

Figure 2. Population data showing the effects of cholinergic and noradrenergic activation on responses and signal-to-noise ratios for whisker stimuli. $A$, Comparison of responses evoked by the PW and four AWs during whisker stimulation at $0.1,2,10$, and $20 \mathrm{~Hz}$. The responses were measured by summing the spikes evoked during a $20 \mathrm{~ms}$ time window after the whisker stimulus during control conditions and during application of carbachol or norepinephrine. The control group $(n=17)$ resulted from averaging the control responses of cells subjected to carbachol and norepinephrine for illustration purposes. However, statistical calculations were performed within subjects by comparing the effect of each drug with its control responses. The two dashed horizontal lines represent the average level of spontaneous firing as spikes per 20 ms for comparison with the whisker-evoked responses. The spontaneous firing (in Hertz) for each condition is also displayed. $\boldsymbol{B}$, Comparison of signal-to-noise ratios of the PW and four AWs during whisker stimulation at $0.1,2,10$, and $20 \mathrm{~Hz}$. Statistical calculations were performed between subjects by comparing the effect of norepinephrine versus the effect of carbachol. Note the much larger signal-to-noise ratios during noradrenergic activation.

control state $(p<0.01)$. The other three AWs during norepinephrine did not change their responses significantly compared with control. Thus, receptive fields are larger during cholinergic than during noradrenergic thalamic activation.

For high-frequency whisker stimulation, during the control state, responses showed strong adaptation (i.e., responses are suppressed by high-frequency whisker stimulation). During carbachol, responses to high-frequency stimulation were significantly enhanced for the PW and all AWs $(p<0.05)$, resulting in a virtual elimination of adaptation (Castro-Alamancos, 2002a) for the PW. During norepinephrine, the response to highfrequency stimulation was significantly enhanced when com- pared with the control state only for the PW ( $p<0.01$ for 10 and $20 \mathrm{~Hz} \mathrm{PW}$ responses). However, the effect of norepinephrine at enhancing high-frequency PW responses was not as strong as that of carbachol; PW high-frequency responses during norepinephrine were significantly smaller than during carbachol $(p<0.05$ carbachol vs norepinephrine for 10 and $20 \mathrm{~Hz}$ PW responses, independent $t$ test). Thus, cholinergic activation is more effective than noradrenergic activation at reducing sensory adaptation in the thalamus.

A measure of the response evoked by a sensory stimulus that considers the saliency of that stimulus with respect to the background noise is the signal-to-noise ratio. The signal-to-noise ratio was calculated by dividing the whisker-evoked response value by the mean spontaneous firing rate for the equivalent $20 \mathrm{~ms}$ period for each cell. Because cells could have no spontaneous activity (e.g., during norepinephrine) or may not respond to a whisker, we substituted zeros with a minimum value (0.0016) for these calculations only. This allowed calculating signal-to-noise ratios for all of the cells, including those that had no spontaneous firing. Figure $2 B$ compares the signal-to-noise ratios during cholinergic and noradrenergic activation. Norepinephrine produced signal-to-noise ratios that were significantly stronger than those of carbachol for all stimulation frequencies of the PW and the first AW and also for low-frequency whisker stimulation of the second AW $(p<0.05)$. Thus, noradrenergic activation in the thalamus leads to significantly higher signal-to-noise ratios than cholinergic activation (Fig. $2 B$ ).

In a previous study, we determined that the enlargement of the receptive fields of VPM cells and increased spontaneous firing caused by carbachol is mediated by muscarinic receptors because the effect is blocked by applying atropine (1-2 mM) in the thalamus (Aguilar and Castro-Alamancos, 2005). Here, we found that the effects of norepinephrine were reversed by the $\alpha$-adrenergic antagonist phentolamine ( $1 \mathrm{~mm} ; n=3)$ applied directly into the thalamus via the microdialysis cannula (supplemental Fig. S1, available at www.jneurosci.org as supplemental material).

\section{Multiwhisker versus single-whisker responses during noradrenergic activation}

During thalamic cholinergic activation, VPM cells respond to a PW and several AWs. However, when the PW and AWs are stimulated simultaneously (multiwhisker stimulation), the response resembles the PW response, as if the AWs had not been stimulated (Aguilar and Castro-Alamancos, 2005). In the present study, we addressed whether noradrenergic activation also lead to differences between single-whisker and multiwhisker stimulation. Figure $3 A$ shows PSTHs comparing the responses of VPM cells $(n=5)$ to simultaneous stimulation of six whiskers with the sum of the responses to each of the six individual whiskers stimulated at low $(0.1 \mathrm{~Hz})$ frequencies. As with cholinergic activation, noradrenergic activation resulted in a sublinear summation of multiwhisker responses; the response to all six whiskers stimulated together was significantly smaller than the sum of the responses to each of the six whiskers $(p<0.001)$. Moreover, when the PSTHs to all six whiskers stimulated together are overlaid with those of the PW whisker alone, they are very similar during both the control state and norepinephrine (Fig. 3B). When these responses are compared (Fig. $3 C$ ), they are not significantly different for low- or high-frequency whisker stimulation. Note that there is a tendency for the response to all six whiskers to be smaller than the PW response; this is attributable to the near absence of long-latency spikes during multiwhisker stimulation (Aguilar and Castro-Alamancos, 2005). Thus, during either cho- 
linergic or noradrenergic thalamic activation, the responses to stimulation of all six whiskers together resemble the responses to stimulation of the PW alone, as if the AWs had not been stimulated.

Effect of cholinergic and noradrenergic activation on corticothalamic responses Up to this point, we described the effects of noradrenergic activation on sensoryevoked responses in VPM cells and compared them with the effects of cholinergic activation. From this point forward, we describe the effects of cholinergic and noradrenergic activation on corticothalamic responses evoked in VPM cells by stimulating the thalamic radiation.

Single-unit recordings were obtained from VPM cells, and a stimulating electrode was placed in the thalamic radiation to evoke corticothalamic responses. A hallmark of the corticothalamic pathway is that it produces strong frequencydependent facilitation (for review, see Castro-Alamancos, 2004b). Accordingly, stimulating and recording electrodes were carefully aligned so that low-intensity stimulation $(<150 \mu \mathrm{A})$ elicited virtually no response to low-frequency stimulation $(0.1 \mathrm{~Hz})$ but a near $100 \%$ firing to stimulation at $10 \mathrm{~Hz}$. Moreover, the responses produced at $10 \mathrm{~Hz}$ were phase-locked to the stimulus and of short latency ( $<7 \mathrm{~ms})$. During the alignment, we purposefully ensured that none of the recorded cells were driven antidromically by the thalamic radiation stimulation at the intensities used. Obviously, other thalamocortical cells, whose axons are coursing closer to the stimulating electrode, are being antidromically discharged. The main consequence of this antidromic discharge in the thalamus will be the recruitment of recurrent (feedback) inhibition from the $\mathrm{nRt}$ via thalamocortical fibers. However, because the nRt is already being recruited by feedforward corticothalamic fibers, this should simply add more of the same, meaning that nRt-mediated inhibition could be enhanced (for a detailed discussion, see Castro-Alamancos, 2004b). Also, the stimulation of thalamocortical axons in the radiation means that action potentials travel to neocortex and stimulate corticothalamic cells via thalamocortical synapses in layer VI. However, this thalamocortical EPSP may arrive in layer VI very close in time with the backpropagating action potential of the stimulated corticothalamic cells, which could impede the generation of additional action potentials because of refractory periods. In conclusion, although these issues must be kept in foresight, we believe that they do not significantly affect the conclusions derived from the present study.

Once the recording and stimulating electrodes had been aligned, the thalamic radiation was stimulated with 10 pulses at different frequencies $(0.1,2,5,10,20$, and $40 \mathrm{~Hz})$ for a minimum of 20 trials for each frequency. This protocol was repeated at least twice for every frequency during control conditions and then again twice after application of the drugs via microdialysis. The spontaneous firing of each cell was measured by counting the number of spikes during the $3 \mathrm{~s}$ period at the beginning of each for the 20 stimulation trials before the corticothalamic stimulation was delivered (i.e., $60 \mathrm{~s}$ period). This value is then converted to either spikes per second (in Hertz) or spikes per $20 \mathrm{~ms}$ for comparison with the whisker-evoked responses. As reported above, carbachol significantly enhanced the spontaneous firing of the cells $(p<0.01 ; n=8)$ (Fig. $4 A$ ). Figure $4 A$ displays population data $(n=8)$ of corticothalamic responses corresponding to the number of spikes evoked during a $20 \mathrm{~ms}$ window after each of the 10 stimuli in a train delivered at different frequencies during control conditions and during application of carbachol in the thalamus. Figure $4 B$ shows population PSTHs of steady-state (10th stimulus in a train) corticothalamic responses before and after application of carbachol into the thalamus. During control (quiescent) conditions, responses to low-frequency stimulation 
A

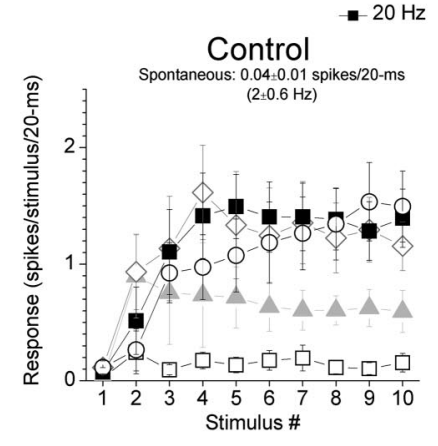

B

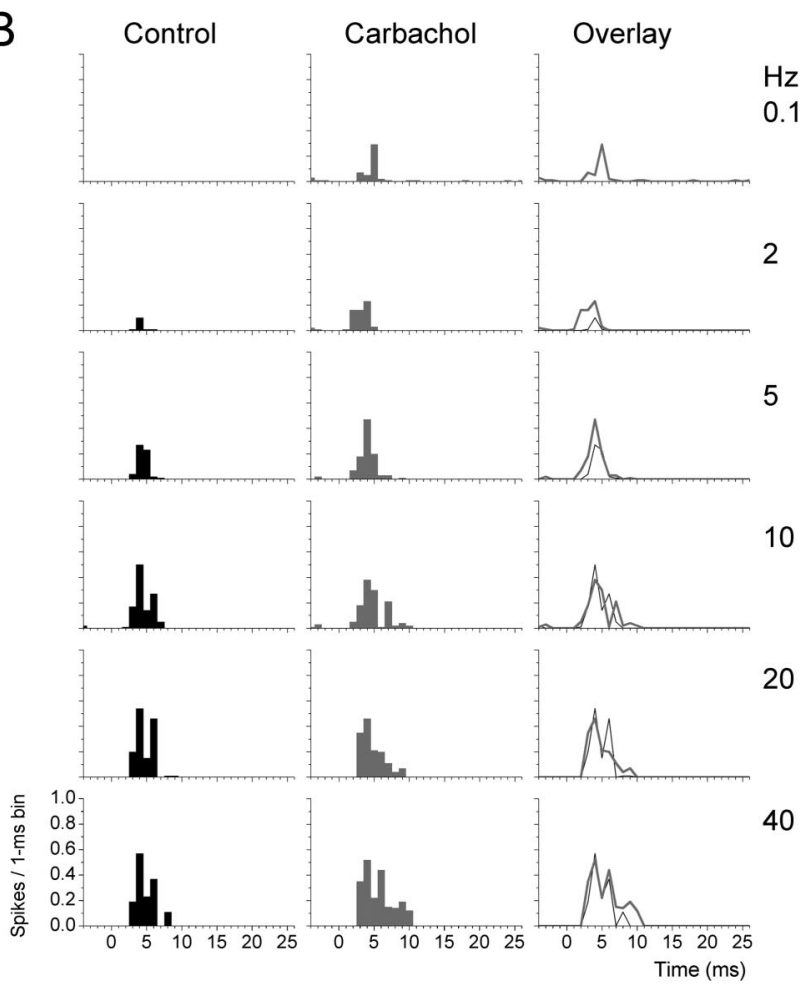

Figure 4. Population data showing the effect of carbachol on corticothalamic responses. $A$, Population corticothalamic responses $(n=8)$ evoked by each of the 10 stimuli in trains at 2,5 , 10,20 , and $40 \mathrm{~Hz}$ during control conditions and during carbachol. Responses were measured by summing the spikes evoked during a $20 \mathrm{~ms}$ time window after each thalamic radiation stimulus. Also displayed is the mean $\pm S D$ level of spontaneous firing during the different conditions, as spikes per $20 \mathrm{~ms}$ and as spikes per second (in Hertz). B, Population PSTHs $(n=8)$ of corticothalamic responses at $0.1,2,5,10,20$, and $40 \mathrm{~Hz}$ during control conditions and during application of carbachol in the thalamus. The PSTHs correspond to the 10th stimulus in the train.

were mostly absent for all cells. As the stimulation frequency increased, corticothalamic responses became apparent, showing strong frequency-dependent facilitation. During application of carbachol into the thalamus, responses to low-frequency stimulation increased significantly ( $p<0.05$ carbachol vs control for 0.1 and $2 \mathrm{~Hz}$ ), whereas high-frequency responses did not change significantly.

In another population of cells, we tested the effects of noradrenergic activation on corticothalamic responses by applying norepinephrine into the thalamus via microdialysis. As reported above, norepinephrine significantly reduced the spontaneous firing of the cells $(p<0.01 ; n=9)$ (Fig. 5A). Figure 5A displays population data $(n=9)$ of corticothalamic responses corresponding to the number of spikes evoked during a $20 \mathrm{~ms}$ window
A
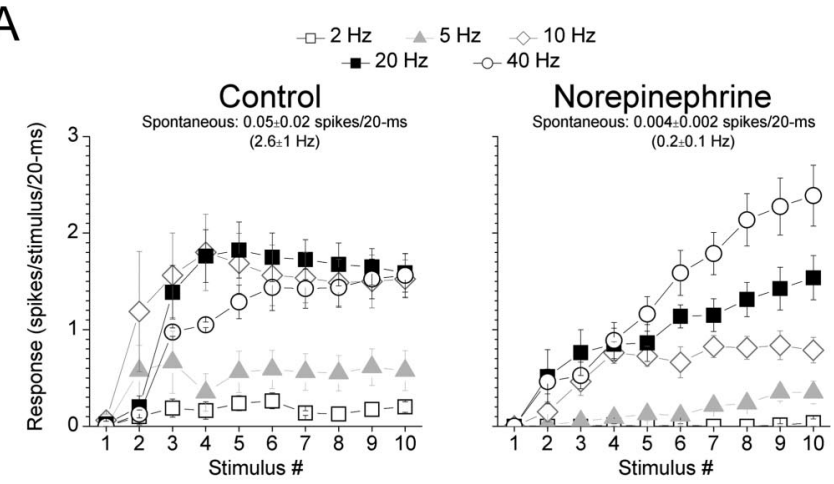

B

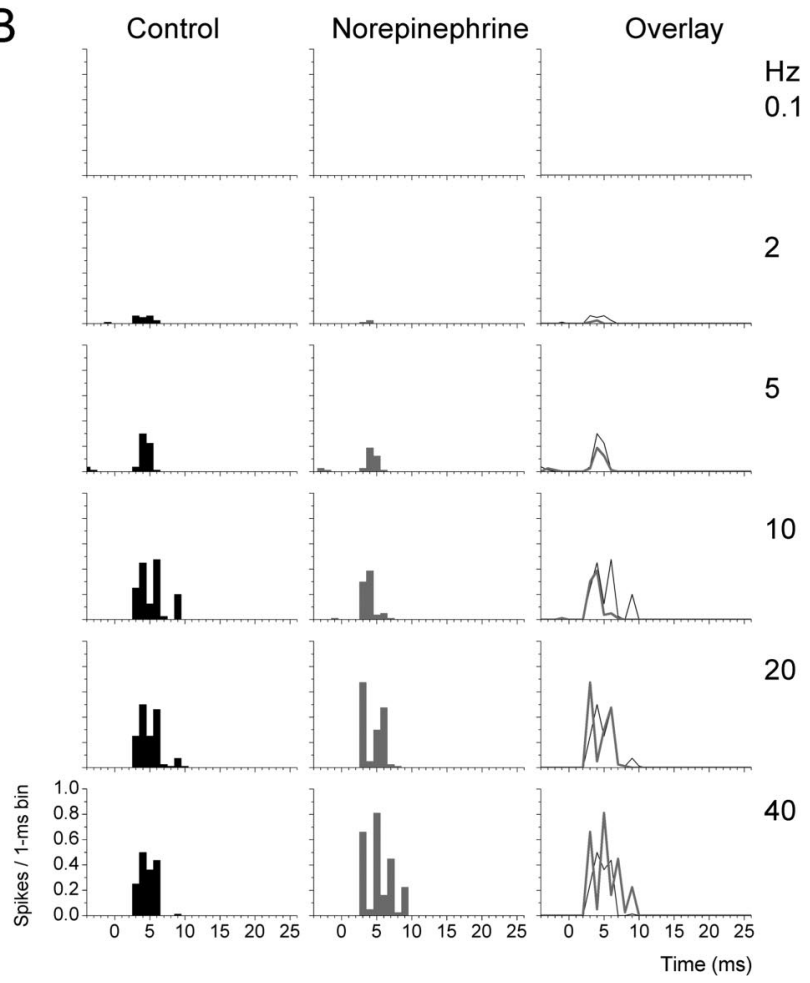

Figure 5. Population data showing the effect of norepinephrine on corticothalamic responses. $\boldsymbol{A}$, Population corticothalamic responses $(n=9)$ evoked by each of the 10 stimuli in trains at 2, 5, 10,20, and $40 \mathrm{~Hz}$ during control conditions and during norepinephrine. Responses were measured by summing the spikes evoked during a $20 \mathrm{~ms}$ time window after each thalamic radiation stimulus. Also displayed is the mean \pm SD level of spontaneous firing during the different conditions, as spikes per $20 \mathrm{~ms}$ and as spikes per second (in Hertz). $\boldsymbol{B}$, Population PSTHs $(n=9)$ of corticothalamic responses at $0.1,2,5,10,20$, and $40 \mathrm{~Hz}$ during control conditions and during application of norepinephrine in the thalamus. The PSTHs correspond to the 10th stimulus in the train.

after each stimulus in a train delivered at different frequencies during control conditions and during application of norepinephrine in the thalamus. Figure $4 B$ shows population PSTHs of steady-state (10th stimulus in a train) corticothalamic responses before and after application of norepinephrine into the thalamus. During application of norepinephrine, responses to lowfrequency stimulation were further suppressed ( $p<0.05$ norepinephrine vs control for 2 and $5 \mathrm{~Hz}$ ), whereas the responses to the highest stimulation frequency $(40 \mathrm{~Hz})$ were significantly enhanced $(p<0.05)$.

Thus, whereas cholinergic activation resulted in an enhancement of low-frequency responses, noradrenergic activation resulted in an additional suppression of low-frequency responses and in the enhancement of high-frequency responses. These ef- 
A
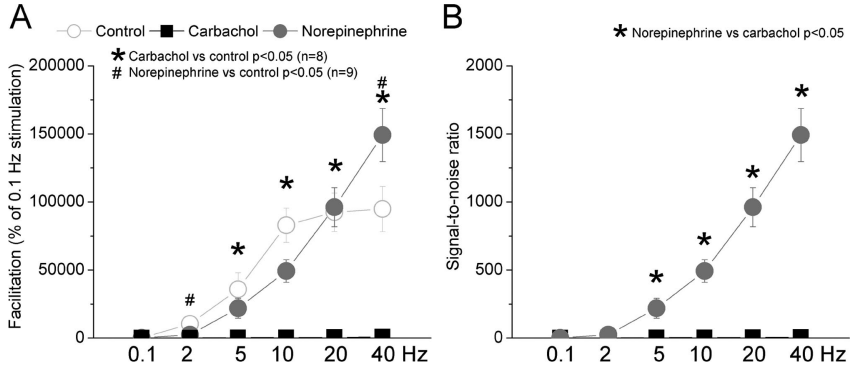

Figure 6. Population data showing the effects of cholinergic and noradrenergic activation on facilitation and signal-to-noise ratios for corticothalamic responses. $\boldsymbol{A}$, Comparison of the amount of facilitation between the different conditions. Facilitation for responses at different frequencies was calculated as the percentage of responses at $0.1 \mathrm{~Hz}$. The responses were measured by summing the spikes evoked during a $20 \mathrm{~ms}$ time window after the thalamic radiation stimulus during control conditions and during application of carbachol or norepinephrine. The control group ( $n=17$ ) resulted from averaging the control responses of cells subjected to carbachol and norepinephrine for illustration purposes. However, statistical calculations were performed within subjects by comparing the effect of each drug with its control responses. $\boldsymbol{B}$ Comparison of signal-to-noise ratios for corticothalamic responses at different frequencies. Statistical calculations were performed between subjects by comparing the effect of norepinephrine versus the effect of carbachol. Note the much larger signal-to-noise ratios during noradrenergic activation.

fects should lead to changes in the amount of facilitation of corticothalamic responses. Indeed, Figure $6 \mathrm{~A}$ plots the amount facilitation during control and either cholinergic or noradrenergic activation. Facilitation was calculated for each cell as the percentage change of the steady-state response (response to the 10th stimulus) as a function of the low-frequency response at $0.1 \mathrm{~Hz}$. As above, to make these calculations, zeros were substituted with a minimum value (0.0016). Also, for illustration purposes only, the control values for cells subjected to carbachol or norepinephrine were averaged together and are displayed in Figure $6 \mathrm{~A}$, but all statistics were computed within subjects. Carbachol resulted in a significant reduction of facilitation for high frequencies $(p<$ $0.01 ; 5,10,20$, and $40 \mathrm{~Hz}$ ). In contrast, norepinephrine resulted in a significant reduction of facilitation for low frequencies $(p<$ $0.05 ; 2 \mathrm{~Hz}$ ) and in the enhancement of facilitation for the highest frequency $(p<0.01 ; 40 \mathrm{~Hz})$.

In addition, we computed signal-to-noise ratios for each cell during cholinergic and noradrenergic activation by dividing the steady-state response for each frequency by the spontaneous firing and compared these values during cholinergic and noradrenergic activation. As shown in Figure $6 B$, during noradrenergic activation in the thalamus, VPM cells have significantly larger signal-to-noise ratios for stimulation frequencies above $5 \mathrm{~Hz}$ than during cholinergic activation (between subjects, $p<0.05$; 5-40 Hz). Thus, noradrenergic activation sharply increases signal-to-noise ratios for high-frequency corticothalamic responses. We also tested whether the effects produced by cholinergic and noradrenergic activation on corticothalamic responses were blocked by specific receptor antagonists (supplemental Fig. S2, available at www.jneurosci.org as supplemental material). The enhancement of VPM cell firing caused by cholinergic activation was blocked by atropine $(n=3)$, whereas the suppression of spontaneous VPM cell firing caused by noradrenergic activation was blocked by phentolamine $(n=3)$. In addition, the results revealed that atropine reversed the effects of carbachol on both facilitation and signal-to-noise ratios, whereas phentolamine reversed the effects of norepinephrine on the signal-tonoise ratios (supplemental Figs. S1, S2, available at www.jneurosci. org as supplemental material).

\section{Effect of cholinergic and noradrenergic activation on $\mathrm{nRt}$ cell firing}

The present results indicate that noradrenergic activation and cholinergic activation produce significantly different effects on sensory and corticothalamic signals to the thalamus. The major difference is that norepinephrine has a strong inhibitory effect on spontaneous firing, AW responses, and low-frequency corticothalamic responses of VPM cells, whereas carbachol has the opposite effects.

The effects of norepinephrine could be caused by an increase in the firing of nRt cells, which would then tonically inhibit VPM cells. To test this possibility, we recorded from nRt cells, which were identifiable by their typical long-lasting responses to sensory stimulation and long burst firing (Hartings et al., 2000, 2003; Castro-Alamancos, 2004b). Interspike interval histograms and autocorrelations for an nRt cell are shown in supplemental Figure S3 (available at www.jneurosci.org as supplemental material). nRt cells strongly enhanced spontaneous firing during noradrenergic activation and suppressed firing during cholinergic activation. An example of the effect of norepinephrine and carbachol application on an nRt cell is shown in Figure $7 A$ (supplemental Fig. S3, available at www.jneurosci.org as supplemental material). During the control condition, the cell fired at $\sim 2.1 \mathrm{~Hz}$, whereas during norepinephrine, the cell firing was $72 \mathrm{~Hz}$. Subsequent application of carbachol to the same cell reduced the spontaneous firing to nil. The suppression of spontaneous firing caused by carbachol was completely reversed by atropine ( $1 \mathrm{mM}$; data not shown). Figure $7 B$ shows an example of the responses of the nRt cell to whisker stimulation delivered at 0.1 and $5 \mathrm{~Hz}$ before and after norepinephrine. Note that, although the spontaneous firing increased, the response to the whisker stimulus was also salient, suggesting that recurrent inhibition was still present on top of the enhanced tonic inhibition. Figure $7 C$ shows population data from nRt cells subjected to either cholinergic or noradrenergic activation. Data from a group of VPM cells are also included for comparison. Norepinephrine significantly enhanced the spontaneous firing of nRt cells but decreased the firing of VPM cells, and carbachol significantly reduced the spontaneous firing of nRt cells while significantly enhancing the firing of VPM cells. Thus, cholinergic and noradrenergic activation has opposite effects on nRt and VPM cells.

These data suggest that, during noradrenergic activation, VPM cells are being inhibited by the robust firing of nRt cells. To test this possibility directly, we recorded from VPM cells and infused $\mathrm{GABA}_{\mathrm{A}}$ and $\mathrm{GABA}_{\mathrm{B}}$ receptor antagonists (BMI and CGP35348, 100-300 $\mu \mathrm{M}$ and 5-10 mM, respectively) directly into the thalamus via microdialysis and then applied norepinephrine. Norepinephrine no longer suppressed the spontaneous firing of VPM cells in the absence of thalamic inhibition. In fact, during disinhibition, norepinephrine significantly enhanced the firing of VPM cells from $3.6 \pm 1.9 \mathrm{~Hz}$ (mean $\pm \mathrm{SD}$ ) to $6.5 \pm 2.9 \mathrm{~Hz}$ (BMI-CGP vs BMI-CGP-norepinephrine; $n=8$ cells; $p<0.05$ ). These results demonstrate that the suppression of spontaneous VPM cell firing produced during norepinephrine is caused by tonic inhibition of VPM cells attributable to increased firing of $\mathrm{nRt}$ cells. Consequently, these results indicate that the enhancement in signal-to-noise ratios characteristic of noradrenergic activation is caused by tonic inhibitory input on VPM cells produced by nRt firing.

\section{Discussion}

Neuromodulators are released in the thalamus during different behavioral states. For example, acetylcholine is released during 


\section{A Spontaneous and drug induced firing of a $\mathrm{nRt}$ cell}

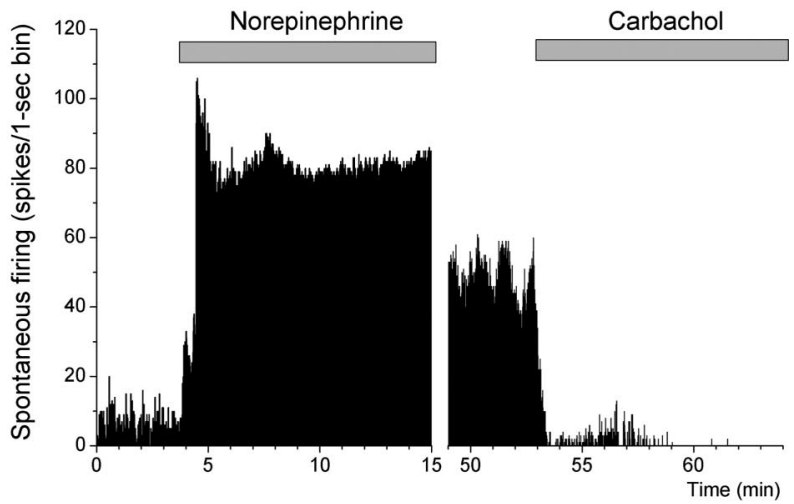

B

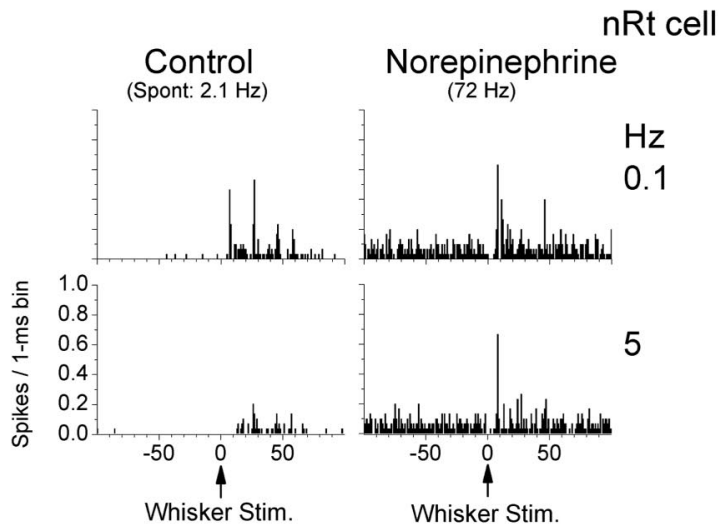

C

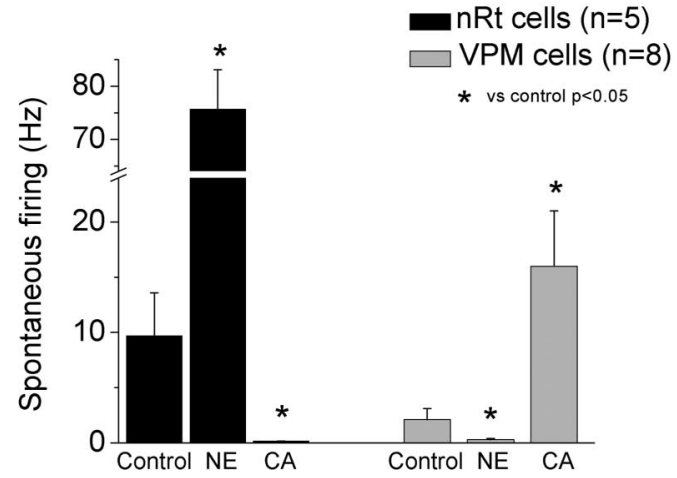

Figure 7. Effects of norepinephrine and carbachol on nRt cells. A, Representative spontaneous activity of an $\mathrm{nRt}$ cell during control conditions and application of norepinephrine. Note that norepinephrine enhanced the spontaneous firing of the nRt cell. Also shown is the effect of application of carbachol before the effect of norepinephrine had subsided. The suppression produced by carbachol is similar to the effects of carbachol on naive nRt cells (not previously subjected to other drugs). $\boldsymbol{B}$, PSTHs showing responses of another nRt cell to stimulation of the PW at 0.1 and $5 \mathrm{~Hz}$ during control conditions and during application of norepinephrine. Also displayed in parentheses is the spontaneous firing of the cell during the different conditions. C, Population data showing the effects of norepinephrine (NE) and carbachol (CA) on the spontaneous firing of (naive) nRt and VPM cells recorded under the same conditions. The control group displayed $(n=13)$ was calculated by averaging the data from all of the cells subjected to carbachol and norepinephrine. Statistical comparisons were within subjects (i.e., between the control and drug conditions for each cell). $\boldsymbol{D}$, Effect of norepinephrine application on the spontaneous firing of VPM cells $(n=7)$ during thalamic disinhibition. Disinhibition was produced by application of BMI-CGP35348 into the thalamus via microdialysis. ${ }^{*} p<0.05$ versus BMI-CGP. paradoxical sleep and during waking, whereas norepinephrine is released during waking in relation to high levels of vigilance and attention. Here we show that noradrenergic activation affects sensory and corticothalamic signals in the whisker thalamus differently than cholinergic activation. For sensory signals, noradrenergic activation sets bottom-up thalamic processing to a focused and noise-free excitatory receptive field, which contrasts with the broad and noisy excitatory receptive field characteristic of cholinergic activation. For corticothalamic signals, noradrenergic activation sets top-down processing to a noise-free highfrequency signal detection mode, whereas cholinergic activation produces a noisy broadband signal detection mode. Apparently, noradrenergic activation sets thalamocortical information processing to a mode that seems more appropriate for sensory discrimination and selective attention.

\section{Noradrenergic activation suppresses spontaneous firing of VPM cells}

Previous work, mostly in slices, revealed the effects of acetylcholine and norepinephrine on thalamic cells (for review, see McCormick, 1992). Thalamocortical cells are depolarized by either acetylcholine or norepinephrine, whereas nRt cells are hyperpolarized by acetylcholine (Ben Ari et al., 1976; McCormick and Prince, 1986; Pinault and Deschenes, 1992) and depolarized by norepinephrine (Kayama et al., 1982; McCormick and Wang, 1991). The depolarizing effect of acetylcholine and norepinephrine on thalamocortical cells is mediated by muscarinic and $\alpha$-adrenergic receptors, respectively, which block a resting $\mathrm{K}^{+}$conductance (McCormick and Prince, 1987, 1988). In addition, activation of $\beta$-adrenergic receptors enhances the hyperpolarization-activated cation current, which also slightly depolarizes thalamocortical cells (McCormick and Pape, 1990). The hyperpolarizing effect of acetylcholine on nRt cells is produced by activation of a $\mathrm{K}^{+}$conductance (McCormick and Prince, 1986), whereas the depolarizing effect of norepinephrine on $\mathrm{nRt}$ cells is mediated by $\alpha$-adrenergic receptors and attributable to a decrease of a resting $\mathrm{K}^{+}$conductance (McCormick and Wang, 1991).

We found that cholinergic activation leads to an increase of VPM cell firing, whereas noradrenergic activation leads to a reduction of VPM cell firing. The effect of cholinergic activation is easily explained by both a direct depolarization of VPM cells and the suppression of nRt cell firing. However, the effect of noradrenergic activation is more complex. It would be explained if the nRt was suppressing VPM cell firing during noradrenergic activation. Indeed, we found that the suppressive effect of norepinephrine on VPM cell firing was completely mediated by the $\mathrm{nRt}$ because, during thalamic disinhibition (block of GABA receptors), norepinephrine no longer suppressed VPM cells. In fact, during disinhibition, VPM cells were excited by norepinephrine. Thus, noradrenergic activation strongly excites nRt cells, which inhibit VPM cells. The differential effects of these neuromodulators on spontaneous firing results in different signal-to-noise ratios in VPM cells (see below).

The considerable differences in spontaneous firing of thalamocortical cells during noradrenergic and cholinergic activation suggests that these neuromodulators may set two different modes of cortical rapid sensory adaptation, because a major determinant of adaptation in the thalamocortical pathway is the firing of thalamocortical cells (Castro-Alamancos and Oldford, 2002; Chung et al., 2002; Castro-Alamancos, 2004a,b). Perhaps cortical sensory adaptation would be reduced during cholinergic activation but present during noradrenergic activation. Future work will need to address this intriguing possibility. 


\section{Compared with cholinergic activation noradrenergic activation focuses receptive fields}

VPM cells respond to deflections of multiple whiskers during light anesthesia (Simons and Carvell, 1989; Armstrong-James and Callahan, 1991; Diamond et al., 1992; Nicolelis and Chapin, 1994; Friedberg et al., 1999; Brecht and Sakmann, 2002; Minnery et al., 2003). Thus, excitatory receptive fields of VPM cells consist of an excitatory center, the PW, and an excitatory surround, the AWs. Cholinergic activation enlarges the excitatory surround of VPM cells (Aguilar and Castro-Alamancos, 2005). In contrast, we found here that noradrenergic activation caused by application of norepinephrine enhanced AW responses but only for one whisker and for low-frequency responses. Consequently, VPM receptive fields are more focused during noradrenergic activation than during cholinergic activation. The selectivity of noradrenergic activation is also present at the temporal level; norepinephrine was less effective than carbachol at facilitating high-frequency responses to whisker stimulation. Whereas carbachol enhanced high-frequency responses for several AWs, norepinephrine only enhanced high-frequency responses for the PW. This indicates that high-frequency sensory inputs are highly focused to the center of the receptive field during noradrenergic activation. The present results demonstrate that different neuromodulators provide the means to dynamically modify receptive field sizes during arousal, from a more focused receptive field characteristic of noradrenergic activation to a broader receptive field typical of cholinergic activation.

When the PW and several AWs are stimulated simultaneously, the response to the PW prevails and AW responses are absent, as if only the center (PW) of the excitatory receptive field is represented during multiwhisker stimulation. This nonlinear summation of responses occurs during both quiescent states and thalamic cholinergic activation (Aguilar and Castro-Alamancos, 2005). In the present study, we found that noradrenergic activation also leads to a nonlinear summation of responses during multiwhisker stimulation. In fact, all response components other than the short-latency PW response are abolished during multiwhisker stimulation in all conditions investigated so far. Therefore, VPM cells represent only the center of the receptive field when center and surround are simultaneously stimulated during either cholinergic or noradrenergic activation.

\section{Noradrenergic activation high-pass filters corticothalamic signals}

In addition to studying how cholinergic and noradrenergic activation affects sensory inputs, we studied how they affect corticothalamic inputs (for a recent review on corticothalamic pathways, see Castro-Alamancos, 2004b). Morphologically and physiologically, VPM cells and upper layer VI corticothalamic cells form closed loops for the flow of information between a thalamic barreloid and a cortical barrel column (Bourassa et al., 1995; Temereanca and Simons, 2004). Corticothalamic synapses display strong frequency-dependent facilitation (Turner and Salt, 1998; Castro-Alamancos and Calcagnotto, 1999, 2001; Turner and Salt, 1999; Golshani et al., 2001), which contrasts with the frequency-dependent depression of primary sensory afferents. Corticothalamic EPSPs are suppressed by both acetylcholine and norepinephrine, an effect that is independent of the postsynaptic actions of these modulators (Castro-Alamancos and Calcagnotto, 2001).

In a previous study, we recorded corticothalamic field potential responses in somatosensory thalamus and found that thalamic activation produced by brainstem reticular formation stimulation led to the high-pass filtering of corticothalamic responses (Castro-Alamancos and Calcagnotto, 2001); lowfrequency responses were suppressed during activated states. This clearly distinguishes corticothalamic from sensory inputs, because sensory inputs drive thalamocortical cells very effectively at low and high frequencies during activated states. Thus, the high-pass filtering is very useful because it ensures that thalamocortical cells are not driven by cortical signals unless those signals arrive at high frequencies.

The present study demonstrates that the high-pass filtering of corticothalamic responses is a selective effect of noradrenergic activation states, which are related to high levels of vigilance and selective attention that should favor sensory relay. In contrast, cholinergic activation augments low-frequency corticothalamic responses, which reduces the amount of facilitation in corticothalamic responses, making VPM cells responsive to a wide frequency band of cortical signals. Hence, during cholinergic activation, the selectivity of VPM cells for high-frequency corticothalamic signals is lost. Certainly, this causes a major problem for thalamocortical sensory processing, because it allows low-frequency cortical signals to become as effective as sensory inputs in driving thalamocortical cells. Such an effect seems undesirable during sensory processing, because thalamocortical cells would not be able to distinguish sensory and cortical inputs.

One possibility is that the enhanced responsiveness to lowfrequency cortical signals during cholinergic activation is related to sensory experiences that are driven by internal, top-down, representations during paradoxical sleep (when cholinergic activation is strong and noradrenergic activation is absent). During paradoxical sleep, cortical cells would be strong drivers of thalamocortical neurons, which may serve to feed top-down representations to upper layers of primary sensory cortex via the thalamus, perhaps related to sensory experiences during this phase of sleep. An important, yet unresolved, issue relates to the firing of corticothalamic cells during behavior. Corticothalamic cells are notorious for being difficult to drive with sensory stimulation (Swadlow and Weyand, 1987; Swadlow, 1990) and have low firing rates even during certain behaviors (Sirota et al., 2005). Hence, it is important to determine when corticothalamic cells fire at high frequencies.

\section{nRt regulates signal-to-noise levels in thalamocortical cells} $n R t$ cells that are responsive to whisker stimulation fire spontaneously in two different modes, a continuous tonic mode and a burst mode (Hartings et al., 2003). Noradrenergic activation produces the continuous tonic firing mode, whereas cholinergic activation suppresses $\mathrm{nRt}$ cell firing. In turn, cholinergic activation enhances the spontaneous firing of VPM cells, causing signal-tonoise ratios to drop, whereas noradrenergic activation reduces the spontaneous firing of VPM cells, causing signal-to-noise ratios to increase sharply. These effects were found to be caused by the firing of $n$ Rt cells because, during block of GABA receptors in the thalamus, norepinephrine no longer suppressed the spontaneous firing of VPM cells or enhanced signal-to-noise ratios for sensory or cortical inputs. In fact, during disinhibition, norepinephrine excited VPM cells. Our results indicate that a primary role of the nRt during sensory processing is to regulate the noise in thalamocortical networks.

An important question related to the effects of norepinephrine is why whisker responses remain in VPM cells if they are strongly inhibited by GABAergic inputs from the nRt. The reason for this seems to be the all-or-none, fast-rising and largeamplitude EPSPs that are triggered by whisker stimulation at 
lemniscal synapses (Castro-Alamancos, 2002a,b), which would overcome the inhibition by nRt. Additional intracellular work is needed to address this question.

\section{Functional implications}

LC cells, the source of norepinephrine to the thalamus, discharge robustly during high vigilance and attentive states, less intensely during waking, and do not fire during sleep, particularly during paradoxical sleep (see Introduction). Thus, noradrenergic activation may provide a dynamic mechanism to (1) focus thalamocortical receptive fields, (2) high-pass filter corticothalamic signals, and (3) enhance signal-to-noise ratios, as cognitive processing demands. We speculate that the more focused receptive fields and signal-to-noise ratios observed during noradrenergic activation compared with cholinergic activation reflect a more appropriate, or at least different, information processing mode for spatial discrimination of sensory inputs, whereas the larger receptive fields, lower signal-to-noise ratios, and broad-frequency spectrum responses to cortical inputs characteristic of cholinergic activation may be related to activated states during paradoxical sleep and nonattentive wakefulness.

\section{References}

Aguilar JR, Castro-Alamancos MA (2005) Spatiotemporal gating of sensory inputs in thalamus during quiescent and activated states. J Neurosci 25:10990-11002.

Armstrong-James M, Callahan CA (1991) Thalamo-cortical processing of vibrissal information in the rat. II. Spatiotemporal convergence in the thalamic ventroposterior medial nucleus (VPm) and its relevance to generation of receptive fields of S1 cortical "barrel" neurones. J Comp Neurol 303:211-224.

Aston-Jones G, Bloom FE (1981) Nonrepinephrine-containing locus coeruleus neurons in behaving rats exhibit pronounced responses to nonnoxious environmental stimuli. J Neurosci 1:887-900.

Aston-Jones G, Chiang C, Alexinsky T (1991) Discharge of noradrenergic locus coeruleus neurons in behaving rats and monkeys suggests a role in vigilance. Prog Brain Res 88:501-520.

Bartho P, Freund TF, Acsady L (2002) Selective GABAergic innervation of thalamic nuclei from zona incerta. Eur J Neurosci 16:999-1014.

Ben Ari Y, Dingledine R, Kanazawa I, Kelly JS (1976) Inhibitory effects of acetylcholine on neurones in the feline nucleus reticularis thalami. J Physiol (Lond) 261:647-671.

Berridge CW, Foote SL (1991) Effects of locus coeruleus activation on electroencephalographic activity in neocortex and hippocampus. J Neurosci 11:3135-3145.

Bourassa J, Pinault D, Deschenes M (1995) Corticothalamic projections from the cortical barrel field to the somatosensory thalamus in rats: a single-fibre study using biocytin as an anterograde tracer. Eur J Neurosci 7:19-30.

Brecht M, Sakmann B (2002) Whisker maps of neuronal subclasses of the rat ventral posterior medial thalamus, identified by whole-cell voltage recording and morphological reconstruction. J Physiol (Lond) 538:495-515.

Castro-Alamancos MA (2002a) Different temporal processing of sensory inputs in the rat thalamus during quiescent and information processing states in vivo. J Physiol (Lond) 539:567-578.

Castro-Alamancos MA (2002b) Properties of primary sensory (lemniscal) synapses in the ventrobasal thalamus and the relay of high-frequency sensory inputs. J Neurophysiol 87:946-953.

Castro-Alamancos MA (2004a) Absence of rapid sensory adaptation in neocortex during information processing states. Neuron 41:455-464.

Castro-Alamancos MA (2004b) Dynamics of sensory thalamocortical synaptic networks during information processing states. Prog Neurobiol 74:213-247.

Castro-Alamancos MA, Calcagnotto ME (1999) Presynaptic long-term potentiation in corticothalamic synapses. J Neurosci 19:9090-9097.

Castro-Alamancos MA, Calcagnotto ME (2001) High-pass filtering of corticothalamic activity by neuromodulators released in the thalamus during arousal: in vitro and in vivo. J Neurophysiol 85:1489-1497.
Castro-Alamancos MA, Oldford E (2002) Cortical sensory suppression during arousal is due to the activity-dependent depression of thalamocortical synapses. J Physiol (Lond) 541:319-331.

Chung S, Li X, Nelson SB (2002) Short-term depression at thalamocortical synapses contributes to rapid adaptation of cortical sensory responses in vivo. Neuron 34:437-446.

Diamond ME, Armstrong-James M, Budway MJ, Ebner FF (1992) Somatic sensory responses in the rostral sector of the posterior group (POm) and in the ventral posterior medial nucleus (VPM) of the rat thalamus: dependence on the barrel field cortex. J Comp Neurol 319:66-84.

el Mansari M, Sakai K, Jouvet M (1989) Unitary characteristics of presumptive cholinergic tegmental neurons during the sleep-waking cycle in freely moving cats. Exp Brain Res 76:519-529.

Foote SL, Aston-Jones G, Bloom FE (1980) Impulse activity of locus coeruleus neurons in awake rats and monkeys is a function of sensory stimulation and arousal. Proc Natl Acad Sci USA 77:3033-3037.

Friedberg MH, Lee SM, Ebner FF (1999) Modulation of receptive field properties of thalamic somatosensory neurons by the depth of anesthesia. J Neurophysiol 81:2243-2252.

Funke K, Pape HC, Eysel UT (1993) Noradrenergic modulation of retinogeniculate transmission in the cat. J Physiol (Lond) 463:169-191.

Golshani P, Liu XB, Jones EG (2001) Differences in quantal amplitude reflect GluR4-subunit number at corticothalamic synapses on two populations of thalamic neurons. Proc Natl Acad Sci USA 98:4172-4177.

Hallanger AE, Levey AI, Lee HJ, Rye DB, Wainer BH (1987) The origins of cholinergic and other subcortical afferents to the thalamus in the rat. J Comp Neurol 262:105-124.

Hartings JA, Temereanca S, Simons DJ (2000) High responsiveness and direction sensitivity of neurons in the rat thalamic reticular nucleus to vibrissa deflections. J Neurophysiol 83:2791-2801.

Hartings JA, Temereanca S, Simons DJ (2003) State-dependent processing of sensory stimuli by thalamic reticular neurons. J Neurosci 23:5264-5271.

Henderson TA, Jacquin M (1995) What makes subcortical barrels? In: Cerebral cortex, Vol 11 (Jones EG, Diamond IT, eds), pp 123-187. New York: Plenum.

Hobson JA, McCarley RW, Wyzinski PW (1975) Sleep cycle oscillation: reciprocal discharge by two brainstem neuronal groups. Science 189:55-58.

Jones BE (1993) The organization of central cholinergic systems and their functional importance in sleep-waking states. Prog Brain Res 98:61-71.

Kayama Y, Negi T, Sugitani M, Iwama K (1982) Effects of locus coeruleus stimulation on neuronal activities of dorsal lateral geniculate nucleus and perigeniculate reticular nucleus of the rat. Neuroscience 7:655-666.

Land PW, Buffer Jr SA, Yaskosky JD (1995) Barreloids in adult rat thalamus: three-dimensional architecture and relationship to somatosensory cortical barrels. J Comp Neurol 355:573-588.

Lindvall O, Bjorklund A, Nobin A, Stenevi U (1974) The adrenergic innervation of the rat thalamus as revealed by the glyoxylic acid fluorescence method. J Comp Neurol 154:317-347.

Livingstone MS, Hubel DH (1981) Effects of sleep and arousal on the processing of visual information in the cat. Nature 291:554-561.

McCormick DA (1992) Neurotransmitter actions in the thalamus and cerebral cortex and their role in neuromodulation of thalamocortical activity. Prog Neurobiol 39:337-388.

McCormick DA, Pape HC (1990) Noradrenergic and serotonergic modulation of a hyperpolarization- activated cation current in thalamic relay neurones. J Physiol (Lond) 431:319-342.

McCormick DA, Prince DA (1986) Acetylcholine induces burst firing in thalamic reticular neurones by activating a potassium conductance. Nature 319:402-405.

McCormick DA, Prince DA (1987) Actions of acetylcholine in the guineapig and cat medial and lateral geniculate nuclei, in vitro. J Physiol (Lond) 392:147-165.

McCormick DA, Prince DA (1988) Noradrenergic modulation of firing pattern in guinea pig and cat thalamic neurons, in vitro. J Neurophysiol 59:978-996.

McCormick DA, Wang Z (1991) Serotonin and noradrenaline excite GABAergic neurones of the guinea-pig and cat nucleus reticularis thalami. J Physiol (Lond) 442:235-255.

Minnery BS, Bruno RM, Simons DJ (2003) Response transformation and receptive field synthesis in the lemniscal trigeminothalamic circuit. J Neurophysiol 90:1556-1570. 
Moruzzi G, Magoun HW (1949) Brain stem reticular formation and activation of the EEG. Electroencephalogr Clin Neurophysiol 1:455-473.

Nicolelis MA, Chapin JK (1994) Spatiotemporal structure of somatosensory responses of many-neuron ensembles in the rat ventral posterior medial nucleus of the thalamus. J Neurosci 14:3511-3532.

Ohara PT, Lieberman AR (1985) The thalamic reticular nucleus of the adult rat: experimental anatomical studies. J Neurocytol 14:365-411.

Paxinos G, Watson C (1982) The rat brain in stereotaxic coordinates. New York: Academic.

Pinault D, Deschenes M (1992) Muscarinic inhibition of reticular thalamic cells by basal forebrain neurones. NeuroReport 3:1101-1104.

Poggio GF, Mountcastle VB (1963) The functional properties of ventrobasal thalamic neurons studied in unanesthetized monkeys. J Neurophysiol 26:775-806.

Rogawski MA, Aghajanian GK (1980a) Activation of lateral geniculate neurons by norepinephrine: mediation by an alpha-adrenergic receptor. Brain Res 182:345-359.

Rogawski MA, Aghajanian GK (1980b) Modulation of lateral geniculate neurone excitability by noradrenaline microiontophoresis or locus coeruleus stimulation. Nature 287:731-734.

Satoh K, Fibiger HC (1986) Cholinergic neurons of the laterodorsal tegmental nucleus: efferent and afferent connections. J Comp Neurol 253:277-302.

Sherman SM, Guillery RW (1996) Functional organization of thalamocortical relays. J Neurophysiol 76:1367-1395.

Simons DJ, Carvell GE (1989) Thalamocortical response transformation in the rat vibrissa/barrel system. J Neurophysiol 61:311-330.

Simpson KL, Altman DW, Wang L, Kirifides ML, Lin RC, Waterhouse BD (1997) Lateralization and functional organization of the locus coeruleus projection to the trigeminal somatosensory pathway in rat. J Comp Neurol 385:135-147.

Sirota MG, Swadlow HA, Beloozerova IN (2005) Three channels of corticothalamic communication during locomotion. J Neurosci 25:5915-5925.

Steriade M, McCarley RW (1990) Brainstem control of wakefulness and sleep. New York: Plenum.
Steriade M, Datta S, Pare D, Oakson G, Curro Dossi RC (1990) Neuronal activities in brain-stem cholinergic nuclei related to tonic activation processes in thalamocortical systems. J Neurosci 10:2541-2559.

Swadlow HA (1990) Efferent neurons and suspected interneurons in S-1 forelimb representation of the awake rabbit: receptive fields and axonal properties. J Neurophysiol 63:1477-1498.

Swadlow HA, Weyand TG (1985) Receptive-field and axonal properties of neurons in the dorsal lateral geniculate nucleus of awake unparalyzed rabbits. J Neurophysiol 54:168-183.

Swadlow HA, Weyand TG (1987) Corticogeniculate neurons, corticotectal neurons, and suspected interneurons in visual cortex of awake rabbits: receptive-field properties, axonal properties, and effects of EEG arousal. J Neurophysiol 57:977-1001.

Temereanca S, Simons DJ (2004) Functional topography of corticothalamic feedback enhances thalamic spatial response tuning in the somatosensory whisker/barrel system. Neuron 41:639-651.

Trageser JC, Keller A (2004) Reducing the uncertainty: gating of peripheral inputs by zona incerta. J Neurosci 24:8911-8915.

Turner JP, Salt TE (1998) Characterization of sensory and corticothalamic excitatory inputs to rat thalamocortical neurones in vitro. J Physiol (Lond) 510:829-843.

Turner JP, Salt TE (1999) Group III metabotropic glutamate receptors control corticothalamic synaptic transmission in the rat thalamus in vitro. J Physiol (Lond) 519:481-491.

Vanderwolf CH (1988) Cerebral activity and behavior: control by central cholinergic and serotonergic systems. Int Rev Neurobiol 30:225-340.

Williams JA, Comisarow J, Day J, Fibiger HC, Reiner PB (1994) Statedependent release of acetylcholine in rat thalamus measured by in vivo microdialysis. J Neurosci 14:5236-5242.

Woolsey TA, Van der Loos H (1970) The structural organization of layer IV in the somatosensory region (SI) of mouse cerebral cortex. The description of a cortical field composed of discrete cytoarchitectonic units. Brain Res 17:205-242. 